\title{
Single and cascaded, magnetically controllable metasurfaces as terahertz filters
}

\author{
Andriy E. Serebryannikov, ${ }^{1, *}$ AKhlesh LaKhtakia, ${ }^{2}$ and Ekmel Ozbay ${ }^{3}$ \\ ${ }^{1}$ Adam Mickiewicz University, Faculty of Physics, 61-614 Poznan, Poland \\ ${ }^{2}$ Pennsylvania State University, Department of Engineering Science and Mechanics, Nanoengineered Metamaterials Group, \\ University Park, Pennsylvania 16802, USA \\ ${ }^{3}$ Bilkent University, Nanotechnology Research Center-NANOTAM, 06800 Ankara, Turkey \\ *Corresponding author: andser@amu.edu.pl
}

Received 26 January 2016; revised 7 March 2016; accepted 10 March 2016; posted 14 March 2016 (Doc. ID 258254); published 7 April 2016

\begin{abstract}
Transmission of a normally incident, linearly polarized, plane wave through either a single electrically thin metasurface comprising $\mathrm{H}$-shaped subwavelength resonating elements made of magnetostatically controllable InAs or a cascade of several such metasurfaces was simulated in the terahertz regime. Stop bands that are either weakly or strongly controllable can be exhibited by a single metasurface by proper choice of the orientation of the magnetostatic field, and a $\sim 19 \%$ downshift of stop bands in the 0.1-5.5 THz spectral regime is possible on increasing the magnetostatic field strength from 0 to $1 \mathrm{~T}$. Better controllability and wider bandwidths are possible by increasing the number of metasurfaces in a cascade, although increase of the total losses can lead to some restrictions. ON/OFF switching regimes, realizable either by applying/removing the magnetostatic field or just by changing its orientation, exist. (c) 2016 Optical Society of America
\end{abstract}

OCIS codes: (050.6624) Subwavelength structures; (120.2440) Filters; (120.7000) Transmission; (160.3820) Magneto-optical materials; (160.3918) Metamaterials.

http://dx.doi.org/10.1364/JOSAB.33.000834

\section{INTRODUCTION}

A planar lattice of identical planar elements is a frequencyselective surface (FSS) $[1,2]$. The elements are usually metallic but can be fabricated of dielectric materials too. Very commonly, FSSs are used in radiofrequency bandpass and bandstop filters. Floquet analysis shows that the transmitted field contains just one propagating plane wave (which is the zeroth-order Floquet harmonic) and numerous evanescent plane waves, when an FSS is illuminated by a plane wave not very obliquely and the lattice parameters are smaller than the free-space wavelength $\lambda_{0}$. Hence, a cascade of multiple identical FSSs that are spaced more than a large fraction of $\lambda_{0}$ apart is not significantly affected by inter-FSS coupling, and the single propagating plane wave can be analyzed using the concept of an equivalent transmission line.

About 10 years ago, researchers began to focus on metasurfaces. Broadly speaking, the interelement spacing is a small fraction of $\lambda_{0}$ and the elemental dimensions normal to the metasurface plane are electrically small [3-5] (i.e., less than about one tenth of the smallest wavelength). The elements need not be periodically arranged [6,7], but usually are [8-10]. Cascading of metasurfaces is also possible [11], although researchers have largely focused on single metasurfaces, which may be considered as two-dimensional (2D) metamaterials.
But cascading either metasurfaces or FSSs closely can yield interesting hybrid regimes [12].

Metasurfaces for the terahertz regime can be fabricated by a host of microfabrication techniques, such as chemical vapor deposition, lithography, and etching [13,14], which has generated considerable experimental research activity [15-17]. Mostly, metasurfaces are fabricated by depositing metallic elements on very thin dielectric sheets. The relative permittivity of the dielectric sheet is a crucial factor when designing a metasurface [18], and its manipulation using quasi-static electric fields [19] and temperature [20], among other agents, provides opportunities for postfabrication dynamic control. Although much less investigated, similar opportunities for postfabrication control are provided by using nonmetallic elements whose constitutive properties can be modified with the aid of temperature [21], quasi-static magnetic fields [22], and other agents.

Cascading of multiple postfabrication-controllable metasurfaces with electrically small inter-metasurface spacing should affect controllability. With this motivation, we started a research program wherein the metasurface elements are made of a doped semiconductor whose relative permittivity can be modulated with a quasi-static magnetic field; each metasurface in a cascade is separated from the one following it by an electrically thin, isotropic, dielectric spacer layer. The number of 


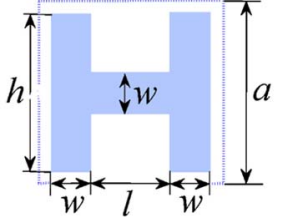

(a)

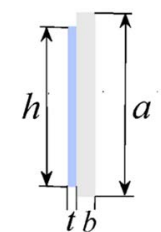

(b)

(c) (d)

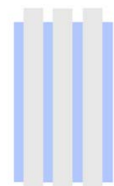

(e)

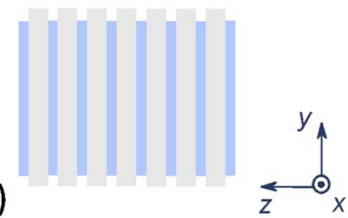

Fig. 1. Schematics of the studied structures: (a) Front view of an $a \times$ $a$ unit cell in a metasurface showing an $\mathrm{H}$-shaped resonating element. Side views for (b) $N=1$, (c) $N=2$, (d) $N=4$, and (e) $N=8$. The Cartesian coordinate axes shown relate to the side views.

metasurfaces in the cascade can be large but the overall cascade thickness must still remain electrically small. Let us note here that magnetostatic tunability of doped semiconductors has a long and continuing history spanning six decades and more [22-26].

This is the first paper emerging from our research program. Our focus lies on the controllability of stop bands in the transmission spectrum of a metasurface cascade in the 0.15.5 THz spectral regime using a quasi-static magnetic field that does not exceed $1 \mathrm{~T}$ in strength. The $\mathrm{H}$-shaped resonating elements are made of InAs [22], and the quasi-static magnetic field can be applied in either the Voigt or the Faraday configuration [27]. The normally incident plane wave is taken to be polarized with one of two mutually orthogonal linear polarization states. The presented results were obtained with the aid of CST Microwave Studio [28], a 3D solver based on the finite integration method.

The plan of this paper is as follows. In Section 2, we present the elemental, metasurface, and cascade dimensions as well as the relative permittivity tensor of InAs, which are important for realizing the desired magnetic-field-controlled tunability. Section 3 elucidates the basic effects of the magnitude and direction of the biasing magnetic field. The obtainable ranges of tunability for polarization-dependent rejection filtering applications are discussed as well. Section 4 is devoted to an understanding of weakening the damping in InAs. Section 5 summarizes the conclusions gleaned from the presented numerical results.

\section{GEOMETRY AND CONSTITUTIVE PROPERTIES}

The studied structure is either a single metasurface $(N=1)$ or a cascade of $N \geq 2$ closely spaced metasurfaces. In each metasurface, $\mathrm{H}$-shaped resonating elements made of a material with relative permittivity tensor $\hat{\epsilon}$ are arranged on a square lattice of side $a$; see Fig. 1(a). Each resonating element is made of two $w \times h$ sections and one $w \times l$ section; the element's thickness is denoted by $t$ in Fig. 1(b). The twofold symmetry of the chosen resonators in the $x y$ plane enables sensitivity to the polarization state of the normally incident plane wave propagating along the

$+z$ axis. When $N=1$, the resonators are printed on a dielectric substrate of thickness $b$ and relative permittivity scalar $\epsilon_{d}$, as shown in Fig. 1(b); when $N>1$, the resonator arrays are separated by dielectric spacers of thickness $b$ and relative permittivity scalar $\epsilon_{d}$, as shown in Figs. 1(c)-1(e). The structure's thickness is denoted by $D=b+t$ for $N=1$ and by $D=$ $(N-1) b+N t$ for $N>1$.

All calculations were made for $\epsilon_{d}=2.1, a=15.56 \mu \mathrm{m}$, $h=14 \mu \mathrm{m}, \quad l=9 \mu \mathrm{m}, \quad w=2.5 \mu \mathrm{m}, \quad t=0.5 \mu \mathrm{m}, \quad$ and $b=2.5 \mu \mathrm{m}$. We assumed that the incident plane wave was either $x$-polarized or $y$-polarized, the incident electric-field phasor $\mathbf{E}_{\text {inc }}$ then being parallel to either the unit vector $\hat{\mathbf{x}}$ (i.e., $\left.\mathbf{E}_{\text {inc }}=\hat{\mathbf{x}} \exp \left(i 2 \pi z / \lambda_{0}\right) \mathrm{Vm}^{-1}\right)$ or the unit vector $\hat{\mathbf{y}}$ (i.e., $\left.\mathbf{E}_{\text {inc }}=\hat{\mathbf{y}} \exp \left(i 2 \pi z / \lambda_{0}\right) \mathrm{Vm}^{-1}\right)$.

InAs is an isotropic dielectric material, but functions as a gyroelectric material when subjected to an external magnetostatic field $\mathbf{B}_{0}$. Along the direction parallel to $\mathbf{B}_{0}$, it always behaves like a Drude metal, regardless of the strength of the applied magnetostatic field.

In the Faraday configuration, $\mathbf{B}_{0}=B_{0} \hat{\mathbf{z}}$ is parallel to the propagation direction of the incident plane wave, and the nonzero components of $\hat{\epsilon}$ in the Cartesian basis are given as functions of the angular frequency $\omega$ as follows [22]:

$$
\begin{gathered}
\epsilon_{x x}=\epsilon_{y y}=\epsilon_{\infty}-\frac{\omega_{p}^{2}\left(\omega^{2}+i \gamma \omega\right)}{\left(\omega^{2}+i \gamma \omega\right)^{2}-\omega^{2} \omega_{c}^{2}}, \\
\epsilon_{x y}=-\epsilon_{y x}=i \frac{\omega \omega_{c} \omega_{p}^{2}}{\left(\omega^{2}+i \gamma \omega\right)^{2}-\omega^{2} \omega_{c}^{2}}, \\
\epsilon_{z z}=\epsilon_{\infty}-\frac{\omega_{p}^{2}}{\left(\omega^{2}+i \gamma \omega\right)} .
\end{gathered}
$$

Here, $\epsilon_{\infty}=16.3$ is the high-frequency relative permittivity, $\gamma / 2 \pi=7.5 \times 10^{11} \mathrm{~Hz}$ is the damping constant, $\omega_{c}=e B_{0} / m^{*}$ is the cyclotron frequency, $\omega_{p}=\sqrt{N e^{2} / \epsilon_{0} m^{*}}$ is the plasma frequency, $N=1.04 \times 10^{23} \mathrm{~m}^{-3}$ is the free-career density at room temperature, $m^{*}=0.004 m_{e}$ is the effective carrier mass, $m_{e}=9.11 \times 10^{-31} \mathrm{~kg}, e=1.6 \times 10^{-19} \mathrm{C}$, and $\epsilon_{0}=$ $8.854 \times 10^{-12} \mathrm{Fm}^{-1}$.

Figure 2 presents $\operatorname{Re}\left(\epsilon_{x x}\right), \operatorname{Re}\left(\epsilon_{z z}\right)$, and $\operatorname{Re}\left(\epsilon_{x y}\right)$ as functions of the frequency $f=\omega / 2 \pi \in[0.1,5.5] \mathrm{THz}$ for six different values of $B_{0} \in[0,1] \mathrm{T}$. The corresponding spectrums of $\operatorname{Im}\left(\epsilon_{x x}\right), \operatorname{Im}\left(\epsilon_{z z}\right)$, and $\operatorname{Im}\left(\epsilon_{x y}\right)$ are shown in Fig. 3. Clearly, one can significantly change the projection of $\hat{\epsilon}$ in the plane perpendicular to the propagation direction of the incident plane wave by varying $B_{0}$. This feature is expected to be an efficient tool to control transmission in a way suitable for filtering applications.

Next, the Voigt configuration can be realized if $\mathbf{B}_{0}$ lies wholly in the $x y$ plane, and is thereby orthogonal to the propagation vector of the incident plane wave. We chose to study two such configurations: (i) Voigt-X wherein $\mathbf{B}_{0}=B_{0} \hat{\mathbf{x}}$ and (ii) Voigt-Y wherein $\mathbf{B}_{0}=B_{0} \hat{\mathbf{y}}$. The nonzero components of $\hat{\epsilon}$ in the Cartesian basis for the Voigt-X configuration are realized by replacing the subscripts $x$ by $y, y$ by $z$, and $z$ by $x$ in Eqs. (1)-(3). Likewise, the nonzero components of $\hat{\epsilon}$ in the Cartesian basis for the Voigt-Y configuration are realized 

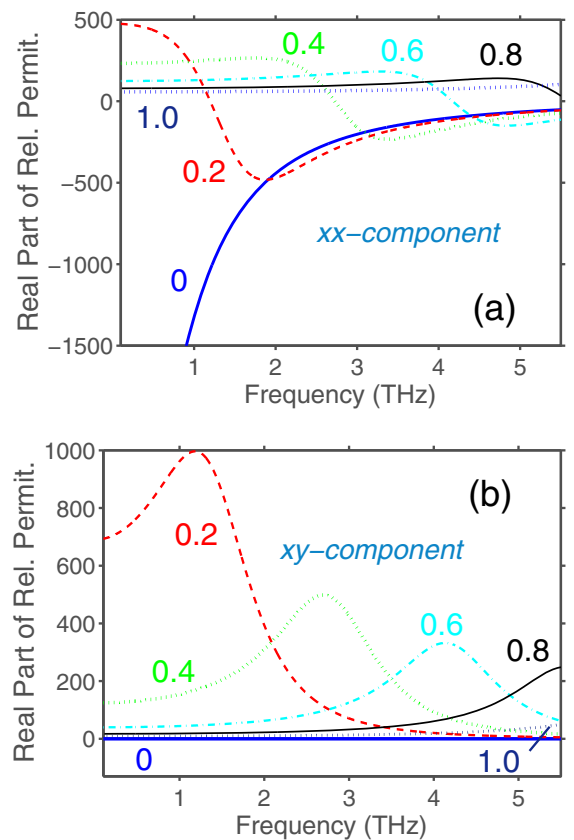

Fig. 2. (a) $\operatorname{Re}\left(\epsilon_{x x}\right)$ and (b) $\operatorname{Re}\left(\epsilon_{x y}\right)$ as functions of the frequency $f \in[0.1,5.5] \mathrm{THz}$ for the Faraday configuration when $B_{0} \in\{0,0.2$, $0.4,0.6,0.8,1\} \mathrm{T}$. The thick solid blue curves are for $B_{0}=0$, the dashed red curves for $B_{0}=0.2 \mathrm{~T}$, the thick dotted green curves for $B_{0}=0.4 \mathrm{~T}$, the dashed-dotted cyan curves for $B_{0}=0.6 \mathrm{~T}$, the thin solid black curves for $B_{0}=0.8 \mathrm{~T}$, and the thin dotted dark-blue curves for $B_{0}=1 \mathrm{~T}$. Note that $\epsilon_{z z}$ defined in Eq. (3) for every value of $B_{0}$ is the same as obtained by setting $B_{0}=0$ in Eq. (1) for $\epsilon_{x x}$.
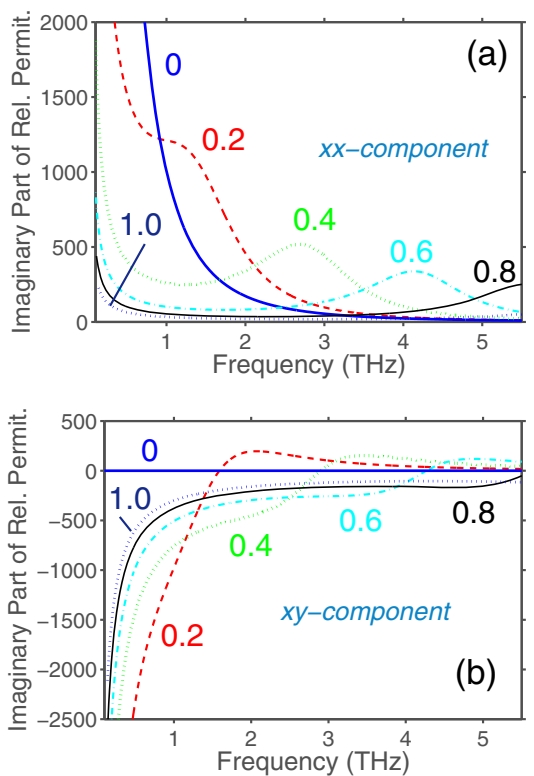

Fig. 3. (a) $\operatorname{Im}\left(\epsilon_{x x}\right)$ and (b) $\operatorname{Im}\left(\epsilon_{x y}\right)$ as functions of the frequency $f \in[0.1,5.5] \mathrm{THz}$ for the Faraday configuration when $B_{0} \in$ $\{0,0.2,0.4,0.6,0.8,1\} \mathrm{T}$. The thick solid blue curves are for $B_{0}=0$, the dashed red curves for $B_{0}=0.2 \mathrm{~T}$, the thick dotted green curves for $B_{0}=0.4 \mathrm{~T}$, the dashed-dotted cyan curves for $B_{0}=0.6 \mathrm{~T}$, the thin solid black curves for $B_{0}=0.8 \mathrm{~T}$, and the thin dotted darkblue curves for $B_{0}=1 \mathrm{~T}$. Note that $\epsilon_{z z}$ defined in Eq. (3) for every value of $B_{0}$ is the same as obtained by setting $B_{0}=0$ in Eq. (1) for $\epsilon_{x x}$. by replacing the subscripts $x$ by $z, z$ by $y$, and $y$ by $x$ in Eqs. (1)-(3).

\section{RESULTS AND DISCUSSION}

Since $a / \lambda_{0} \simeq 0.29$ at $5.5 \mathrm{THz}$, the transmitted field contains just one propagating plane wave and numerous evanescent plane waves. The propagating plane wave is specular, but the evanescent plane waves are nonspecular. The electric-field phasor of the specularly transmitted plane wave is given by $\mathbf{E}_{\mathrm{tr}}^{\text {spec }}=\left(\tau_{x x} \hat{\mathbf{x}}+\right.$ $\left.\tau_{y x} \hat{\mathbf{y}}\right) \exp \left(i 2 \pi z / \lambda_{0}\right)$ when the incident electric-field phasor is $x$-polarized, and by $\mathbf{E}_{\mathrm{tr}}^{\text {spec }}=\left(\tau_{x y} \hat{\mathbf{x}}+\tau_{y y} \hat{\mathbf{y}}\right) \exp \left(i 2 \pi z / \lambda_{0}\right)$ when the incident electric-field phasor is $y$-polarized. We computed the copolarized specular-transmission coefficients $\tau_{x x}$ and $\tau_{y y}$ as well as the cross-polarized specular-transmission coefficients $\tau_{x y}$ and $\tau_{y x}$ as functions of frequency $f$ and the number of metasurfaces $N$.

\section{A. $N=1$ and $N=2$}

Computed spectrums of $\left|\tau_{x x}\right|$ and $\left|\tau_{y y}\right|$ presented in Fig. 4 for $B_{0} \in\{0,1\}$ T and all three configurations demonstrate the basic effects of the applied magnetostatic field for the solitary metasurface (i.e., $N=1$ ) shown in Fig. 1(b). Cross-polarization specular transmission is certainly possible in the Faraday configuration but is so weak as to be negligible. Also, reversal of the propagation direction of the incident plane wave has very weak effects and was therefore ignored for this paper.

Figure 4 contains two prominent stop bands, the first with its center frequency between 1.4 and $1.9 \mathrm{THz}$ in Fig. 4(a), and the second with its center frequency between 3 and $3.6 \mathrm{THz}$ in Fig. $4(\mathrm{~b})$. The first stop band occurs for the $x$-polarized
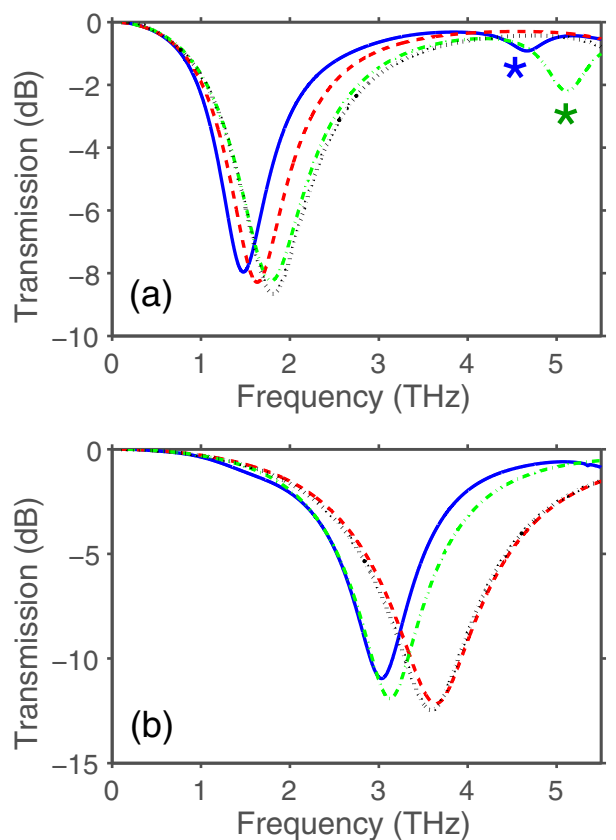

Fig. 4. Computed spectrums of (a) $\left|\tau_{x x}\right|$ and (b) $\left|\tau_{y y}\right|$ for $B_{0} \in\{0,1\} \mathrm{T}$ and $N=1$; see Fig. 1(b). The solid blue curves are for the Faraday configuration with $B_{0}=1 \mathrm{~T}$, the dashed-dotted green curves for the Voigt-X configuration with $B_{0}=1 \mathrm{~T}$, the dashed red curves for the Voigt-Y configuration with $B_{0}=1 \mathrm{~T}$, and the dotted black curves for $B_{0}=0$. See the text for explanations of the asterisks. 

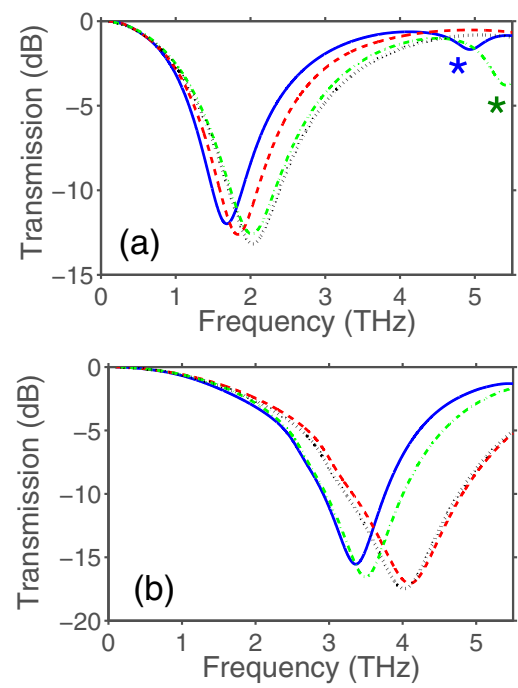

Fig. 5. Computed spectrums of (a) $\left|\tau_{x x}\right|$ and (b) $\left|\tau_{y y}\right|$ for $B_{0} \in\{0,1\} \mathrm{T}$ and $N=2$; see Fig. 1(c). The solid blue curves are for the Faraday configuration with $B_{0}=1 \mathrm{~T}$, the dashed-dotted green curves for the Voigt-X configuration with $B_{0}=1 \mathrm{~T}$, the dashed red curves for the Voigt-Y configuration with $B_{0}=1 \mathrm{~T}$, and the dotted black curves for $B_{0}=0$. See the text for explanations of the asterisks.

incident wave. The center frequency of this stop band is negligibly sensitive to $B_{0}$ in the Voigt-X configuration, significantly sensitive in the Voigt- $Y$ configuration, and even more sensitive in the Faraday configuration. The second stop band occurs for the $y$-polarized incident wave. The center frequency of this stop band is negligibly sensitive to $B_{0}$ in the Voigt-Y configuration, significantly sensitive in the Voigt-X configuration, and still more sensitive in the Faraday configuration.

Thus, both stop bands redshift for the Faraday configuration, regardless of the polarization state of the incident plane wave. However, a similar redshift of one stop band takes place in the Voigt configuration only for $\mathbf{E}_{\text {inc }} \perp \mathbf{B}_{0}$-for the first stop band when $\mathbf{E}_{\text {inc }} \| \hat{\mathbf{x}}$, and for the second stop band when $\mathbf{E}_{\text {inc }} \| \hat{\mathbf{y}}$.

With $D=3 \mu \mathrm{m}$ being the total thickness of the structure for $N=1$, it is noteworthy that $D / \lambda_{0}=1.48 \times 10^{-2}$ at $f=$ $1.48 \mathrm{THz}$ (the dip of $\left|\tau_{x x}\right|$ for the Faraday configuration at $B_{0}=1 \mathrm{~T}$ ) and $D / \lambda_{0}=3.64 \times 10^{-2}$ at $f=3.64 \mathrm{THz}$ (the dip of $\left|\tau_{y y}\right|$ for the Voigt-Y configuration at $B_{0}=1 \mathrm{~T}$ ). Thus, the entire structure is electrically thin, which is desirable in filters.
Let us add an identical metasurface at the front side of the initial structure, as shown in Fig. 1(c). Now, the two metasurfaces can be coupled. Spectrums of $\left|\tau_{x x}\right|$ and $\left|\tau_{y y}\right|$ are presented in Fig. 5 for $B_{0} \in\{0,1\}$ T and all three configurations. A general trend is the enhancement of the stop bands for $N=2$ as compared to $N=1$, with the deepening of the transmission dips requiring the expense of fabricating another metasurface.

The two prominent stop bands in Fig. 5 are blueshifted in comparison to their counterparts in Fig. 4. As examples,

(i) the first stop band for the Faraday configuration is centered at $f=1.48 \mathrm{THz}$ for $N=1$ but at $f=1.68 \mathrm{THz}$ for $N=2$, and

(ii) the second stop band for the Voigt-Y configuration is centered at $f=3.64 \mathrm{THz}$ for $N=1$ but at $f=$ $4.10 \mathrm{THz}$ for $N=2$,

when $B_{0}=1 \mathrm{~T}$. Capacitative coupling between the two metasurfaces in Fig. 1(c) may be responsible for these blueshifts. Other characteristics related to the sensitivity to $B_{0}$ for the various configurations are the same for $N=2$ as for $N=1$.

Additionally, Figs. 4(a) and 5(a) indicate the signatures of new stop bands in the Faraday and Voigt-X configurations in the neighborhood of $f=5 \mathrm{THz}$, when the magnetostatic field is switched on. These stop bands are identified by asterisks in both figures. However, the transmission dips are weakly pronounced, so that the new stop bands are likely to be of limited interest.

With $D=3.5 \mu \mathrm{m}$ being the total thickness of the structure for $N=2$, it is noteworthy that $D / \lambda_{0} \approx 1.96 \times 10^{-2}$ at $f=$ $1.68 \mathrm{THz}$ (the dip of $\left|\tau_{x x}\right|$ for the Faraday configuration at $B_{0}=1 \mathrm{~T}$ ) and $D / \lambda_{0} \approx 4.78 \times 10^{-2}$ at $f=4.10 \mathrm{THz}$ (the dip of $\left|\tau_{y y}\right|$ for the Voigt-Y configuration at $\left.B_{0}=1 \mathrm{~T}\right)$. Thus, the entire structure remains electrically thin for $N=2$.

To demonstrate the magnetostatic tunability of the chosen structures, Tables 1 and 2 present the center frequencies of the first and second stop bands in relation to $B_{0}$ for the Faraday, Voigt-X, and Voigt-Y configurations, when $N=1$ and $N=2$, respectively. Calculations were made for $B_{0} \in\{0,0.2,0.4,0.6,0.8,1.0,2.0\} \mathrm{T}$; results are reported for $B_{0}=2 \mathrm{~T}$ rather for the purpose of comparison, because such a strong magnetostatic field can be quite impractical in physical situations.

Tables 1 and 2 confirm that the magnetostatic tunability of the first and the second stop bands is weak when $\mathbf{B}_{0} \| \mathbf{E}_{\text {inc }}$ (which can happen only in the two Voigt configurations).

Table 1. Center Frequencies (THz) of the First and Second Stop Bands in Relation to $B_{0}$ for the Faraday, Voigt-X, and Voigt-Y Configurations, When $N=1$

\begin{tabular}{|c|c|c|c|c|c|c|}
\hline \multirow[b]{2}{*}{$B_{0}(T)$} & \multicolumn{2}{|c|}{ Faraday } & \multicolumn{2}{|c|}{ Voigt-X } & \multicolumn{2}{|c|}{ Voigt-Y } \\
\hline & $\left|\tau_{x x}\right|$ & $\left|\tau_{y y}\right|$ & $\left|\tau_{x x}\right|$ & $\left|\tau_{y y}\right|$ & $\left|\tau_{x x}\right|$ & $\left|\tau_{y y}\right|$ \\
\hline 0 & 1.81 & 3.59 & 1.81 & 3.59 & 1.81 & 3.59 \\
\hline 0.2 & 1.80 & 3.59 & 1.82 & 3.60 & 1.81 & 3.62 \\
\hline 0.4 & 1.75 & 3.51 & 1.82 & 3.54 & 1.79 & 3.64 \\
\hline 0.6 & 1.67 & 3.37 & 1.82 & 3.43 & 1.75 & 3.65 \\
\hline 0.8 & 1.58 & 3.21 & 1.81 & 3.30 & 1.69 & 3.64 \\
\hline 1.0 & 1.48 & 3.03 & 1.79 & 3.13 & 1.63 & 3.64 \\
\hline 2.0 & 1.02 & 2.17 & 1.64 & 2.34 & 1.29 & 3.58 \\
\hline
\end{tabular}


Table 2. Center Frequencies (THz) of the First and Second Stop Bands in Relation to $B_{0}$ for the Faraday, Voigt-X, and Voigt-Y Configurations, When $N=2$

\begin{tabular}{|c|c|c|c|c|c|c|}
\hline \multirow[b]{2}{*}{$B_{0}(T)$} & \multicolumn{2}{|c|}{ Faraday } & \multicolumn{2}{|c|}{ Voigt-X } & \multicolumn{2}{|c|}{ Voigt-Y } \\
\hline & $\left|\tau_{x x}\right|$ & $\left|\tau_{y y}\right|$ & $\left|\tau_{x x}\right|$ & $\left|\tau_{y y}\right|$ & $\left|\tau_{x x}\right|$ & $\left|\tau_{y y}\right|$ \\
\hline 0 & 2.03 & 4.02 & 2.03 & 4.02 & 2.03 & 4.02 \\
\hline 0.2 & 2.03 & 4.03 & 2.05 & 4.03 & 2.03 & 4.07 \\
\hline 0.4 & 1.98 & 3.94 & 2.05 & 3.97 & 2.01 & 4.09 \\
\hline 0.6 & 1.90 & 3.78 & 2.05 & 3.85 & 1.97 & 4.10 \\
\hline 0.8 & 1.80 & 3.58 & 2.4 & 3.69 & 1.91 & 4.10 \\
\hline 1.0 & 1.68 & 3.36 & 2.02 & 3.50 & 1.83 & 4.10 \\
\hline 2.0 & 1.17 & 2.39 & 1.84 & 2.58 & 1.44 & 4.02 \\
\hline
\end{tabular}

The magnetostatic tunability is very poor for $B_{0} \lesssim 0.2 \mathrm{~T}$ but quite strong and monotonic for $B_{0} \gtrsim 0.2 \mathrm{~T}$, for (i) the Faraday configuration regardless of the orientation $\mathbf{E}_{\text {inc }}$, (ii) the Voigt-X configuration when $\mathbf{E}_{\text {inc }} \| \hat{\mathbf{y}}$, and (iii) the Voigt-Y configuration when $\mathbf{E}_{\text {inc }} \| \hat{\mathbf{x}}$. The shift of the center frequency becomes larger as $B_{0}$ increases beyond $\sim 0.4 \mathrm{~T}$ for these two cases, and one may choose $0.3 \mathrm{~T}$ or thereabouts as the minimal value of $B_{0}$, starting from which efficient tunability can be achieved.

While increasing $B_{0}$ from 0 to $1 \mathrm{~T}$ with just one metasurface (i.e., $N=1$ ), the first stop band can be tuned over a range of $320 \mathrm{GHz}$ for the Faraday configuration and $180 \mathrm{GHz}$ for the Voigt-Y configuration, and the second stop band over a range of $560 \mathrm{GHz}$ for the Faraday configuration and $470 \mathrm{GHz}$ for the Voigt-X configuration. Hence, the second stop band is more sensitive to the variations in $B_{0}$ than the first stop band, whereas the Faraday configuration leads to a wider tunability range than the Voigt configurations.

Even wider tunability ranges would be available with magnetostatic fields of higher strength. For instance, $\tau_{x x}$ for the Voigt-X configuration becomes sensitive to variations in $B_{0}>1 \mathrm{~T}$. Hence, a possible reason for low sensitivity at $B_{0} \leq 1 \mathrm{~T}$ is that the magnetostatic field is not sufficiently strong. However, $\tau_{y y}$ for the Voigt-Y configuration remains rather weakly sensitive to variations in $B_{0} \leq 2 \mathrm{~T}$, but may become strongly sensitive for $B_{0}>2 \mathrm{~T}$.

Comparing Table 1 with Table 2, one can see that the only significant differences are that the stop bands blueshift and the tunability range is somewhat enhanced for $N=2$ in comparison to $N=1$. When $N=2$, the first stop band can be tuned over a range of $350 \mathrm{GHz}$ for the Faraday configuration and $200 \mathrm{GHz}$ for the Voigt-Y configuration, and the second stop band over a range of $670 \mathrm{GHz}$ for the Faraday configuration and $530 \mathrm{GHz}$ for the Voigt-X configuration, as $B_{0}$ increases from 0 to $1 \mathrm{~T}$. Hence, adding the second metasurface allows us to extend the tunability range of the second stop band by up to $19 \%$.

\section{B. $N=4$ and $N=8$}

Increasing $N$ to 4 , we next discuss transmission through the structure shown in Fig. 1(d). The general effect of cascading is the formation of alternating passbands and stop bands that are very pronounced, as is known for one-dimensional periodic multilayers [29], two-dimensional photonic crystals [30], and metamaterials [12]. At the same time, absorption loss increases with $N$, leading to some practical restrictions.
Computed spectrums of $\left|\tau_{x x}\right|$ and $\left|\tau_{y y}\right|$ for $B_{0} \in\{0,1\}$ T are presented in Fig. 6. As expected, the stop bands are deeper than their counterparts in Figs. 4 and 5. Application of $B_{0}=1 \mathrm{~T}$ in the Faraday configuration results in narrowing and redshifting of the stop bands arising at $B_{0}=0$, for both polarization states of the incident plane wave. Application of $B_{0}=1 \mathrm{~T}$ in the Voigt-X (resp. Voigt-Y) configuration results in the same effect only for the second (resp. first) stop band. The magnetostatically induced stop bands identified by asterisks for $B_{0}=1 \mathrm{~T}$ and the Faraday as well as the Voigt-X configurations in Figs. 4(a) and 5(a) also appear in Fig. 6(a), and are also identified by asterisks.

Next, ON/OFF switching (with a high contrast in transmission) is possible. For example, $\left|\tau_{y y}\right|=-2.7 \mathrm{~dB}$ in Fig. 6(b) for the Faraday configuration at $B_{0}=1 \mathrm{~T}(\mathrm{ON})$, whereas $\left|\tau_{y y}\right|=$ $-12 \mathrm{~dB}$ at $B_{0}=0$ (OFF), when $f=5.3 \mathrm{THz}$. Such ON/ OFF switching is possible in some broad spectral regimes, e.g., in the vicinity of $f=5 \mathrm{GHz}$-indicated by a square
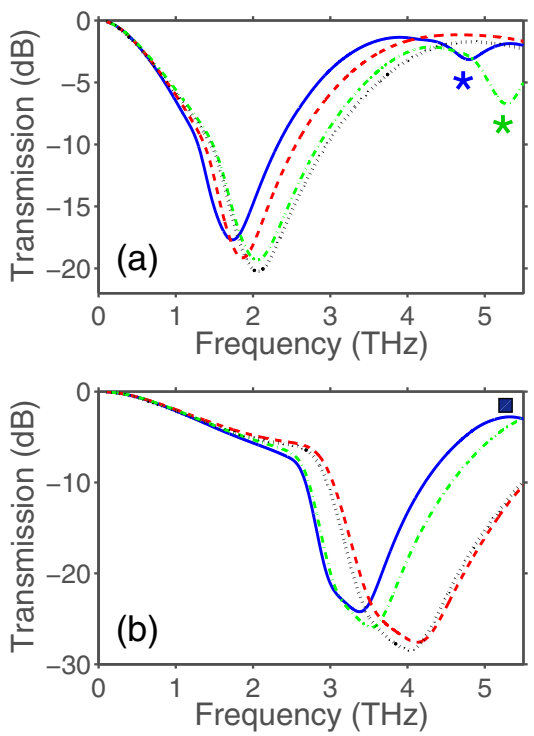

Fig. 6. Computed spectrums of (a) $\left|\tau_{x x}\right|$ and (b) $\left|\tau_{y y}\right|$ for $B_{0} \in$ $\{0,1\} \mathrm{T}$ and $N=4$; see Fig. $1(\mathrm{~d})$. The solid blue curves are for the Faraday configuration with $B_{0}=1 \mathrm{~T}$, the dashed-dotted green curves for the Voigt-X configuration with $B_{0}=1 \mathrm{~T}$, the dashed red curves for the Voigt-Y configuration with $B_{0}=1 \mathrm{~T}$, and the dotted black curves for $B_{0}=0$. See the text for explanations of the asterisks and the square. 
at the top in Fig. 6(b). To obtain stronger transmission in the ON state, optimization of the structure is required.

At $f=5.5 \mathrm{THz}, D / \lambda_{0}=0.17$ when $N=4$, and $D / \lambda_{0}=0.39$ when $N=8$; thus, even the structure depicted in Fig. $1(\mathrm{e})$ is still less than half a free-space wavelength in thickness. The question arises: Does the doubling of the number of coupled metasurfaces from 4 to 8 have desirable consequences?

Figure 7 presents the computed spectrums of $\left|\tau_{x x}\right|$ and $\left|\tau_{y y}\right|$ for $B_{0} \in\{0,1\}$ T and $N=8$. All the basic effects of the magnetostatic field gleaned from Figs. 4-6 are still present; very importantly, wide and deep stop bands are finally formed. The bandwidths of the first stop band at the $-20 \mathrm{~dB}$ level are $1 \mathrm{THz}$ for the Faraday configuration and $1.4 \mathrm{THz}$ for $B_{0}=0$ in Fig. 7, whereas the bandwidths of the second stop band at the same level are $1.6 \mathrm{THz}$ and $2.6 \mathrm{THz}$, respectively. Comparison with Fig. 6(b) shows that doubling the number of metasurfaces can lead to significant deepening and widening of the stop bands.

The switching option provided by Fig. 6 for $N=4$ is available also for $N=8$, as indicated by the square in Fig. 7(b). For example, $\left|\tau_{y y}\right|=-6.5 \mathrm{~dB}$ at $f=5.2 \mathrm{THz}$ for the Faraday configuration with $B_{0}=1 \mathrm{~T}$, whereas $\left|\tau_{y y}\right|=-25 \mathrm{~dB}$ when $B_{0}=0$. Thus, transmission efficiency in the $\mathrm{ON}$ state decreases due to the enhancement of absorption loss with higher $N$. Generally speaking, a suitable value of $N$ will have to be chosen, depending on the specific requirements for the operating regime(s) and available fabrication facilities.

Figures 6(a) and 7(a) together suggest that another type of ON/OFF switching is possible. For instance, in Fig. 7(a) $\left|\tau_{x x}\right|=-2.2 \mathrm{~dB}$ in the Voigt-Y configuration and $\left|\tau_{x x}\right|=$ $-13 \mathrm{~dB}$ in the Voigt-X configuration, at $f=5.2 \mathrm{THz}$ when
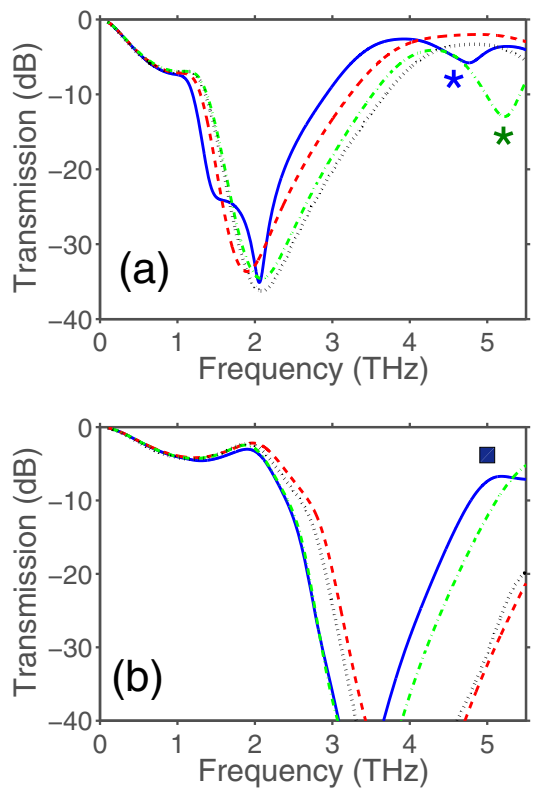

Fig. 7. Computed spectrums of (a) $\left|\tau_{x x}\right|$ and (b) $\left|\tau_{y y}\right|$ for $B_{0} \in$ $\{0,1\} \mathrm{T}$ and $N=8$; see Fig. $1(\mathrm{e})$. The solid blue curves are for the Faraday configuration with $B_{0}=1 \mathrm{~T}$, the dashed-dotted green curves for the Voigt-X configuration with $B_{0}=1 \mathrm{~T}$, the dashed red curves for the Voigt-Y configuration with $B_{0}=1 \mathrm{~T}$, and the dotted black curves for $B_{0}=0$. The asterisks and the square denote the same as in Fig. 6.
$B_{0}=1 \mathrm{~T}$. Thus, $\mathbf{B}_{0}=1 \hat{\mathbf{y}} \mathrm{T}$ in the ON state, but $\mathbf{B}_{0}=1 \hat{\mathbf{x}} \mathrm{T}$ in the OFF state, while the frequency and the polarization state of the incident plane wave remain unchanged.

\section{WEAK DAMPING}

Suppose that the damping is weakened, possibly using a composite material comprising InAs and a gain material [31-33]. As a detailed analysis lies outside the scope of this paper, we set $\gamma / 2 \pi=1.19 \times 10^{11} \mathrm{THz}$ to obtain illustrative results.

Figure 8 presents the transmission results for $N=2$. The dips are at least 50\% deeper and slightly blueshifted than their counterparts in Fig. 5. A well-pronounced dip arising at $f=5.42 \mathrm{THz}$ in the Voigt-X configuration should be noticed, at which $\left|\tau_{x x}\right|=-12 \mathrm{~dB}$. This dip is well suited for ON/OFF switching, because transmission is high for the Faraday and Voigt-Y configurations when $B_{0}=1 \mathrm{~T}$, as well as for $B_{0}=0\left(\left|\tau_{x x}\right|>-0.35 \mathrm{~dB}\right)$. Hence, one has the freedom to use either the Faraday or the Voigt-Y configuration with $B_{0}=1 \mathrm{~T}$ or simply set $B_{0}=0$ for the $\mathrm{ON}$ state, whereas the Voigt-X configuration should be used for the OFF state.

Figure 9 presents the results for $N=8$. Comparing with Fig. 7, one should note larger depth and steeper boundaries of the stop bands. Furthermore, a deep stop band for the Voigt-X configuration is centered at $f=5.27 \mathrm{THz}$, with the ON/OFF contrast even higher than in the similar case in Fig. 8(a). In particular, $\left|\tau_{x x}\right|=-31 \mathrm{~dB}(\mathrm{OFF})$ at the dip for the Voigt-X configuration when $B_{0}=1 \mathrm{~T}$, whereas $\left|\tau_{x x}\right|>$ $-2.5 \mathrm{~dB}(\mathrm{ON})$ for $B_{0}=0$ as well as for the Faraday and the Voigt-Y configurations with $B_{0}=1 \mathrm{~T}$. The flexibility for the ON state is achieved here due to weaker damping, as is clear from comparison with Fig. 7(a).

Furthermore, wideband and efficient ON/OFF switching can be obtained in the case indicated by the square in Fig. 9(b). At $f=5.1 \mathrm{THz},\left|\tau_{y y}\right|=-2.5 \mathrm{~dB}(\mathrm{ON})$ for the Faraday configuration when $B_{0}=1 \mathrm{~T}$, whereas $\left|\tau_{y y}\right|=-27.5 \mathrm{~dB}(\mathrm{OFF})$ for $B_{0}=0$. Alternatively, the Voigt-X configuration can be
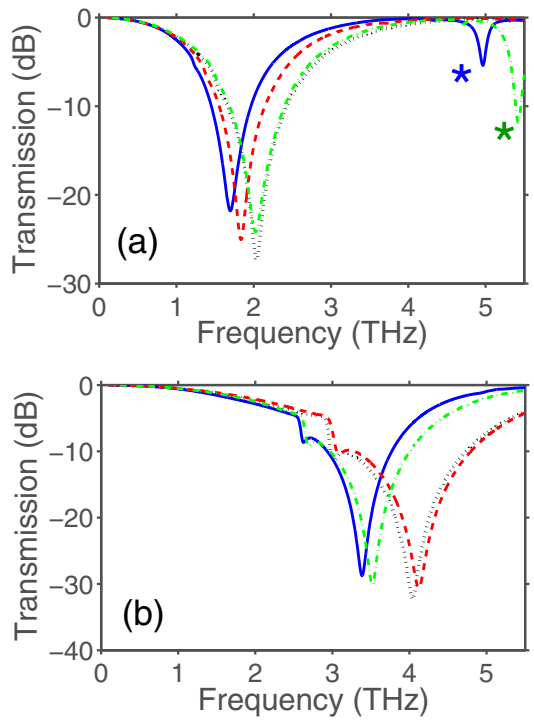

Fig. 8. Same as Fig. 5 but for $\gamma / 2 \pi=1.19 \times 10^{11} \mathrm{THz}$. 

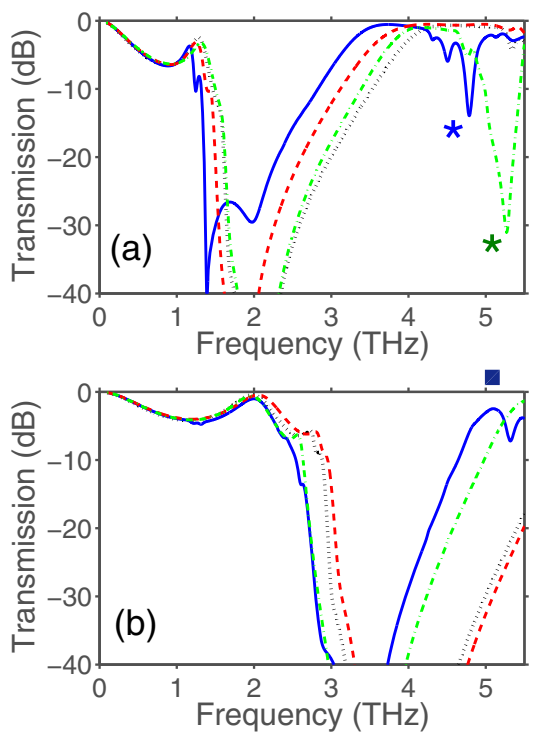

Fig. 9. Same as Fig. 7 but for $\gamma / 2 \pi=1.19 \times 10^{11} \mathrm{THz}$.

utilized to obtain the OFF state. Clearly, this switching modality would be more desirable than the similar one indicated by the square in Fig. $7(\mathrm{~b})$, because larger $\left|\tau_{y y}\right|$ can be obtained in the ON state.

Proper parameter adjustment may allow ON/OFF switching for both $x$ - and $y$-polarized plane waves simultaneously, owing to the close proximity of the asterisk and the square on the frequency axes, as is clear from Fig. 9. In addition, it is expected that the same frequency could be used for the both incident polarization states, while transmission in the $\mathrm{ON}$ state is quite high.

One more switching modality should be mentioned (not shown). In this case, switching between two adjacent stop bands can be obtained simply by changing the linear polarization state of the incident plane wave to the one that is orthogonal, provided that the direction and the magnitude of $\mathbf{B}_{0}$ are properly chosen. For example, this can be done in the Faraday configuration at $B_{0}=1 \mathrm{~T}$ in the vicinity of $f=2.7 \mathrm{THz}$ when $N=4$, while smaller and larger values of $N$ are less preferable. Let us note the existence of polarization transformers in the terahertz regime [34].

\section{CONCLUDING REMARKS}

Transmission of a normally incident, linearly polarized plane wave through either a single metasurface comprising $\mathrm{H}$-shaped subwavelength resonating elements made of InAs or a cascade of several such metasurfaces was simulated with the finite integration method implemented using a commercial 3D solver. The metasurfaces are electrically thin, and even the thickest cascade considered did not exceed half of the free-space wavelength in thickness.

The permittivity of InAs in the terahertz regime can be significantly affected by applying a relatively weak magnetostatic field. This property leads to a strong modification of the transmission spectrum that is characterized by shifts of stop bands relative to their spectral locations when the magnetostatic field has been switched off.

Six basic combinations of the orientations of the magnetostatic field and the incident electric field were examined. The magnetostatic field is oriented normally to the metasurface(s) in the Faraday configuration, and the magnetostatically controllable spectral shifts occur regardless of the polarization state of the incident plane waves. In either of the two Voigt configurations, the magnetostatic field is oriented wholly tangential to the metasurface(s), and a specific stop band will be highly affected for one polarization state of the incident plane wave and weakly affected for the orthogonal polarization state, depending on whether the incident electric field is parallel or perpendicular to the magnetostatic field.

The ranges of magnetostatic tunability of the stop bands in the $0.1-5.5 \mathrm{THz}$ spectral regime were determined for each of the six basic combinations of the orientations of the magnetostatic field and the incident electric field. The largest relative shift-from 3.59 to $3.03 \mathrm{THz}$ as the magnetostatic field increased from 0.2 to $1.0 \mathrm{~T}$-was found to occur with a single $(N=1)$ metasurface, when the magnetostatic field is oriented normally to the metasurface and the incident electric field is oriented parallel to the two identical legs of each H-shaped resonating element. A somewhat smaller relative shift was found when the magnetostatic field is oriented parallel to the central segment and the incident electric field is oriented parallel to the two legs of each $\mathrm{H}$-shaped resonating element.

The tunability range can be extended by $9 \%-19 \%$ by having a cascade of two closely spaced, identical, and parallel metasurfaces. Stop bands with rather large depth and width that increase with the number of metasurfaces were found for cascades of four and eight metasurfaces. Even eight cascaded metasurfaces together remain subwavelength in thickness in the entire frequency range considered; a single metasurface on a substrate can be just $0.025 \lambda_{0}$ thick.

Additionally, ON/OFF switching regimes_realizable either by applying/removing the magnetostatic field or just by changing its orientation - exist. In principle, such switching is possible for two mutually orthogonal, linearly polarized plane waves, independently of each other. Strategies to weaken damping are important, because the use of materials with similar characteristics as InAs but with weaker damping promises a wider variety of switching modalities as well as better performance. Switching between the two adjacent stop bands, one for $x$-polarized and the other for $y$-polarized incident plane waves, is possible by cascading only four metasurfaces. Weaker damping facilitates switching with fewer metasurfaces in the cascade.

Magnetostatic tunability can also be displayed by individual metasurfaces and metasurface cascades fabricated by depositing metallic resonating elements on magnetostatically sensitive substrates. However, the deposition of magnetostatically sensitive resonating elements on negligibly absorbing dielectric substrates, as studied in this paper, should be preferable for the transmission outside stop bands to be as high as possible. We plan to conduct a comparative study.

Funding. North Atlantic Treaty Organization (SET-193); Devlet Planlama Örgütü (DPT) (FOTON, HAMIT); 
TUBITAK (109A015, 109E301, 113E331); Charles Godfrey Binder Endowment at Penn State; Turkish Academy of Sciences; National Science Centre, Poland (MagnoWa DEC-2-12/07/E/ST3/00538, MetaSel DEC-2015/17/B/ ST3/00118).

Acknowledgment. AL thanks the Charles Godfrey Binder Endowment at Penn State for ongoing support of his research. EO acknowledges partial support from the Turkish Academy of Sciences.

\section{REFERENCES}

1. T.-K. Wu, Frequency Selective Surfaces (Wiley, 1995).

2. B. A. Munk, Frequency Selective Surfaces: Theory and Design (Wiley, 2000).

3. C. L. Holloway, E. R. Kuester, and D. R. Novotny, "Waveguides composed of metafilms/metasurfaces: the two-dimensional equivalent of metamaterials," IEEE Antennas Wireless Propag. Lett. 8, 525-529 (2009).

4. C. Fietz and G. Shvets, "Homogenization theory for simple metamaterials modeled as one-dimensional arrays of thin polarizable sheets," Phys. Rev. B 82, 205128 (2010).

5. S. Walia, C. M. Shah, P. Gutruf, H. Nili, D. R. Chowdhury, W. Withayachumnankul, M. Bhaskaran, and S. Sriram, "Flexible metasurfaces and metamaterials: a review of materials and fabrication processes at micro- and nano-scales," Appl. Phys. Rev. 2, 011303 (2015).

6. C. Pfeiffer and A. Grbic, "Metamaterial Huygens surfaces: tailoring wave fronts with reflectionless sheets," Phys. Rev. Lett. 110, 197401 (2013).

7. F. Aieta, A. Kabiri, P. Genevet, N. Yu, M. A. Kats, Z. Gaburro, and F. Capasso, "Reflection and refraction of light from metasurfaces with phase discontinuities," J. Nanophoton. 6, 063532 (2012).

8. I. B. Vendik, O. G. Vendik, M. A. Odit, D. V. Kholodnyak, S. P. Zubko, M. F. Sitnikova, P. A. Turalchuk, K. N. Zemlyakov, I. V. Munina, D. S. Kozlov, V. M. Turgaliev, A. B. Ustinov, Y. Park, J. Kihm, and C.-W. Lee, "Tunable metamaterials for controlling $\mathrm{THz}$ radiation," IEEE Trans. Terahertz Sci. Technol. 2, 538-549 (2012).

9. Y. Wen, W. Ma, J. Bailey, G. Matmon, and X. Yu, "Broadband terahertz metamaterial absorber based on asymmetric resonators with perfect absorption," IEEE Trans. Terahertz Sci. Technol. 5, 406411 (2015)

10. M. Veysi, C. Guclu, O. Boyraz, and F. Capolino, "Thin anisotropic metasurfaces for simultaneous light focusing and polarization manipulation," J. Opt. Soc. Am. B 32, 318-323 (2015).

11. J. Chen and H. Mosallaei, "Truly achromatic optical metasurfaces: a filter circuit theory-based design," J. Opt. Soc. Am. B 32, 2115-2121 (2015).

12. M. Beruete, P. Rodriguez-Ulibarri, V. Pacheco-Peña, M. Navarro-Cía, and A. E. Serebriyannikov, "Frozen mode from hybridized extraordinary transmission and Fabry-Perot resonances," Phys. Rev. B 87, 205128 (2013).

13. S. Franssila, Introduction to Microfabrication, 2nd ed. (Wiley, 2010).
14. R. J. Martín-Palma and A. Lakhtakia, Nanotechnology (SPIE, 2010).

15. L. Huang, D. R. Chowdhury, S. Ramani, M. T. Reiten, S.-N. Luo, A. J. Taylor, and H.-T. Chen, "Experimental demonstration of terahertz metamaterial absorbers with a broad and flat high absorption band," Opt. Lett. 37, 154-156 (2012).

16. C. Jansen, I. A. I. Al-Naib, N. Born, and M. Koch, "Terahertz metasurfaces with high Q-factors," Appl. Phys. Lett. 98, 051109 (2011).

17. S.-W. Qu, W.-W. Wu, B.-J. Chen, H. Yi, X. Bai, K. B. Ng, and C. H. Chan, "Controlling dispersion characteristics of terahertz metasurface," Sci. Rep. 5, 9367 (2015).

18. X. Xia, Y. Sun, H. Yang, H. Feng, L. Wang, and C. Gua, "The influences of substrate and metal properties on the magnetic response of metamaterials at terahertz region," J. Appl. Phys. 104, 033505 (2008).

19. M. Decker, C. Kremers, A. Minovich, I. Staude, A. E. Miroshnichenko, D. Chigrin, D. N. Neshev, C. Jagadish, and Y. S. Kivshar, "Electrooptical switching by liquid-crystal controlled metasurfaces," Opt. Express 21, 8879-8885 (2013).

20. R. Singh, A. K. Azad, Q. X. Jia, A. J. Taylor, and H.-T. Chen, "Thermal tunability in terahertz metamaterials fabricated on strontium titanate single-crystal substrates," Opt. Lett. 36, 1230-1232 (2011).

21. J. Han and A. Lakhtakia, "Semiconductor split-ring resonators for thermally tunable terahertz metamaterials," J. Mod. Opt. 56, 554-557 (2009).

22. J. Han, A. Lakhtakia, and C.-W. Qiu, "Terahertz metamaterials with semiconductor split-ring resonators for magnetostatic tunability," Opt. Express 16, 14390-14396 (2008).

23. V. I. Fistul', Heavily Doped Semiconductors (Springer, 1969).

24. R. F. Wallis, J. J. Brion, E. Burstein, and A. Hartstein, "Theory of surface polaritons in anisotropic dielectric media with application to surface magnetoplasmons in semiconductors," Phys. Rev. B 9, 3424-3437 (1974).

25. M. Shalaby, M. Peccianti, Y. Ozturk, and R. Morandotti, "A magnetic non-reciprocal isolator for broadband terahertz operation," Nat. Commun. 4, 1558 (2013)

26. S. Chen, F. Fan, X. He, M. Chen, and S. Chang, "Multifunctional magneto-metasurface for terahertz one-way transmission and magnetic field sensing," Appl. Opt. 54, 9177-9182 (2015).

27. O. Madelung, Introduction to Solid-State Theory (Springer, 1978).

28. CST-Computer Simulation Technology, https://www.cst.com (accessed 29 Oct., 2015).

29. H. A. MacLeod, Thin-Film Optical Filters, 4th ed. (CRC Press, 2010).

30. K. Sakoda, Optical Properties of Photonic Crystals (Springer, 2001).

31. S. Wuestner, A. Pusch, K. L. Tsakmakidis, J. M. Hamm, and O. Hess, "Overcoming losses with gain in a negative refractive index metamaterial," Phys. Rev. Lett. 105, 127401 (2010).

32. T. G. Mackay and A. Lakhtakia, "Dynamically controllable anisotropic metamaterials with simultaneous attenuation and amplification," Phys. Rev. A 92, 053847 (2015).

33. I. V. Shadrivov, M. Lapine, and Y. S. Kivshar, eds., Nonlinear, Tunable and Active Metamaterials (Springer, 2015).

34. L. Cong, W. Cao, Z. Tian, J. Gu, J. Han, and W. Zhang, "Manipulating polarization states of terahertz radiation using metamaterials," New J. Phys. 14, 115013 (2012). 